\title{
Lipedema and the Evolution to Lymphedema With the Progression of Obesity
}

Lívia Maria Pereira de Godoy ${ }^{1}$, Henrique Jose Pereira de Godoy ${ }^{1}$, Paula Pereira de Godoy Capeletto ${ }^{2}$, Maria de Fatima Guerreiro Godoy ${ }^{3}$, Jose Maria Pereira de Godoy 4,5

1. General Practice, Clínica Godoy, São José do Rio Preto, BRA 2. General Practice, Clínica Godoy, Sao Jose do Rio Preto, BRA 3. Medicine, São José do Rio Preto School of Medicine (FAMERP), São José do Rio Preto, BRA 4. Cardiology and Cardiovascular Surgery, São José do Rio Preto School of Medicine (FAMERP), Sao Jose do Rio Preto, BRA 5. Angiology and Vascular Surgery, Clínica Godoy, Sao Jose do Rio Preto, BRA

Corresponding author: Jose Maria Pereira de Godoy, godoyjmp@gmail.com

\section{Abstract}

Aim: The aim of the present study was to evaluate the prevalence of subclinical and clinical systemic lymphedema in patients with lipedema and different body mass index (BMI) values.

Method: A cross-sectional study was conducted to determine the prevalence of subclinical systemic lymphedema and clinical lymphedema of the lower limbs detected by bioimpedance (InBody S10 device, Seoul, Korea) in 258 women with clinically diagnosed lipedema. The patients were divided into three groups based on BMI: Group I - BMI below $30 \mathrm{~kg} / \mathrm{m}^{2}$; Group II - BMI between 30 and $40 \mathrm{~kg} / \mathrm{m}^{2}$; and Group III - BMI 40 to $50 \mathrm{~kg} / \mathrm{m}^{2}$.

Results: Fisher's exact test revealed a statistically significant difference between Group I and both Groups II and III ( $\mathrm{p}=0.0001)$ regarding the occurrence of lower limb lymphedema.

Conclusion: Patients with lipedema can develop edema even when their weight is within the standards of normality. However, obesity is an aggravating factor, as the prevalence of lipedema increases progressively with the increase in weight.

Review began 11/14/2020 Review ended 11/22/2020 Published 12/02/2020

๑) Copyright 2020 Pereira de Godoy et al. This is an open access article distributed under the terms of the Creative Commons Attribution License CC-BY 4.0., which permits unrestricted use, distribution, and reproduction in any medium, provided the original author and source are credited.
Categories: Dermatology, Physical Medicine \& Rehabilitation, Other

Keywords: lipedema, lymphedema, obesity, bioimpedance

\section{Introduction}

Lipedema was first described by Allen and Hines in 1940 as a clinical condition associated with the enlargement of the extremities [1]. Individuals with this condition exhibit greater redistribution of fatty tissue to the extremities, which leads to enlargement of the limb [2]. The physiopathological processes are not well defined, but changes in the lymphatic system and fatty tissue are the most cited [2-8]. Polygenic combinations of hormonal, microvascular, and lymphatic disorders may be partially related to the development of lipedema [9]. Some surgical options are reported for select cases, such as liposuction and excisional lipectomy [2].

In one study, the lipedema group had a larger area of adipocytes in comparison to the control group and a similar area to that found in obese controls and obese individuals with lipedema. The number of macrophages increased significantly in the skin and fat in the lipedema group compared to the control group. There was also an increase in dermal vessels in the non-obese lipedema group compared to the control group [10].

The psychological effects of the increase in the volume of the limb and pain are the aspects that most impact the quality of life of these patients [11]. Recent studies describe a novel concept of lymphedema associated with obesity evaluated using bioelectrical impedance analysis. In an initial phase, an increase in intracellular and extracellular liquids is detected in all extremities and the trunk without reaching the stage of clinical lymphedema. This initial stage is denominated subclinical systemic lymphedema. When reading the clinical stage in all extremities and the trunk, the patient has clinical systemic lymphedema [11-14].

The present study aimed to evaluate the prevalence of subclinical and clinical systemic lymphedema in patients with lipedema and different body mass index (BMI) values.

\section{Materials And Methods}

\section{Patients and setting}

We evaluated 258 women with a clinical diagnosis of lipedema at the Clínica Godoy in 2019. 


\section{Design}

A cross-sectional study was conducted to determine the prevalence of subclinical systemic lymphedema and clinical lymphedema of the lower limbs detected by bioimpedance (InBody S10 device, Seoul, Korea) in 258 women with clinically diagnosed lipedema.

\section{Inclusion criteria}

All women at the clinic for the treatment of stage I and II varicose veins and a diagnosis of associated lipedema.

\section{Exclusion criteria}

Women with other causes of clinically diagnosed edema, such as heart failure, kidney failure, and hypoproteinemia.

\section{Development}

All patients who met the inclusion criteria were consecutively submitted to bioelectrical impedance analysis for the investigation of subclinical systemic lymphedema, which is characterized by intracellular and extracellular liquids as well as liquids of the lower limb, upper limbs and trunk above normal values. The diagnosis of lower limb lymphedema was based on bioimpedance criteria. The patients were divided into three groups based on BMI: Group I - BMI below $30 \mathrm{~kg} / \mathrm{m}^{2}$; Group II - BMI between 30 and $40 \mathrm{~kg} / \mathrm{m}^{2}$; and Group III - BMI 40 to $50 \mathrm{~kg} / \mathrm{m}^{2}$. Subclinical systemic lymphedema and clinical lower limb lymphedema were investigated in each participant. This study received approval from the Human Research Ethics Committee of the São José do Rio Preto School of Medicine, São Paulo, Brazil (\#2.928.922).

\section{Results}

Two hundred fifty-eight consecutive women with lipedema were evaluated using the InBody S10 bioimpedance device: 98 in Group I (BMI less than $30 \mathrm{~kg} / \mathrm{m}^{2}$ ), 124 in Group II (BMI between 30 and 40 $\mathrm{kg} / \mathrm{m}^{2}$ ), and 36 in Group III (BMI between 40 and $50 \mathrm{~kg} / \mathrm{m}^{2}$ ). Among the 98 women in Group I, 16 (16.3\%) had subclinical systemic lymphedema (SSL). Moreover, 60 of the 124 women in Group II (48.3\%) and 26 of the 36 women in Group III (72.2\%) had SSL. Fisher's exact test revealed a statistically significant difference among the groups regarding the occurrence of SSL $(p=0.0001)$. A significant difference was also found between Groups II and III $(\mathrm{p}=0.001)$.

Clinical lymphedema of the lower limbs was detected in six of the 98 women in Group I (6.1\%), 64 of the 124 women in Group II (51.6\%), and 28 of the 36 women in Group III (77.8\%). Fisher's exact test revealed a statistically significant difference between Group I and both Groups II and III $(p=0.0001)$ regarding the occurrence of lower limb lymphedema. A significant difference was also found between Groups II and III ( $\mathrm{p}$ = $0.001)$.

\section{Discussion}

The present study shows that obesity is an aggravating factor of edema, as edema was progressively frequent with the increase in BMI among women with lipedema. Moreover, approximately $16 \%$ of the women with a BMI lower than $30 \mathrm{~kg} / \mathrm{m}^{2}$ had subclinical systemic lymphedema. This finding suggests that normal fatty tissue in non-obese women with lipedema exhibits important changes associated with the development of edema. However, obesity is an aggravating factor. This is the first study to offer such information.

A previous study found no significant change in the expression of genes associated with inflammation in adipose-derived stem cells (ASCs) in lipedema or differentiated adipocytes [10]. These data suggest that there is no inflammatory process that may cause the edema, at least not in non-obese individuals. Thus, there must be other physiopathological processes.

The vascular changes in microcirculation, especially lymphatic vessels, suggest lymphostasis [4,5,8,15]. A study involving lymphoscintigraphy demonstrated lymphatic alterations in lipedema, suggesting lymphedema resulting from the lymphatic microcirculation [15]. The treatment of these patients using lymphatic drainage results in a reduction of the symptoms and circumference of the limb, which confirms the existence of the lymphatic component [9].

Other conditions associated with edema, such as idiopathic cyclic edema and cellulite, are common in these women. According to a study in the publication phase, $10 \%$ have idiopathic cyclic edema. Moreover, cellulite is found in more than $50 \%$ of women. Therefore, these are two aggravating factors generally associated with edema $[9,16]$. Another study detected an increase in the sodium content of adipose tissue in patients with lipedema [17]. Thus, multiple factors are involved in the formation of edema, but all processes lead to an overload of the lymphatic system. 
Another important aspect of lipedema is the greater potential for adipogenic differentiation in comparison to patients without this condition [10]. This aspect suggests greater facility in the development of obesity. Thus, the most important advice for these patients is not to gain weight, thereby avoiding the addition of another physiopathological process.

Obesity is the main aggravating factor of edema in these patients, but serious harm can occur in the lymphatic system, leading to overload and the formation of lymphedema. In terms of treatment, the combination of effective lymphatic drainage techniques and prophylactic weight loss to avoid the aggravation of the condition is the best therapeutic approach.

A perspective to be analyzed in future research would be to have a control group with patients without the presence of lipedema and other types of edema. Also, evaluating therapeutic responses in the short and long term will provide us with greater parameters for the clinical application and treatment of these patients.

\section{Conclusions}

Patients with lipedema can develop edema even when their weight is within the standards of normality. However, obesity is an aggravating factor, as the prevalence of lipedema increases progressively with the increase in weight. Therefore, it is suggested to assess whether weight loss alone reduces this edema.

\section{Additional Information \\ Disclosures}

Human subjects: Consent was obtained by all participants in this study. Human Research Ethics Committee of the São José do Rio Preto School of Medicine (FAMERP) issued approval \#2.928.922. Animal subjects: All authors have confirmed that this study did not involve animal subjects or tissue. Conflicts of interest: In compliance with the ICMJE uniform disclosure form, all authors declare the following: Payment/services info: All authors have declared that no financial support was received from any organization for the submitted work. Financial relationships: All authors have declared that they have no financial relationships at present or within the previous three years with any organizations that might have an interest in the submitted work. Other relationships: All authors have declared that there are no other relationships or activities that could appear to have influenced the submitted work.

\section{References}

1. Wold LE, Hines EA Jr, Allen EV: Lipedema of the legs; a syndrome characterized by fat legs and edema . Ann Intern Med. 1951, 34:1243-1250. 10.7326/0003-4819-34-5-1243

2. Redondo Galán C, García Bascones M, Marquina Valero MA: Lipoedema: symptoms, diagnosis and treatment. A literature review. (Article in Spanish). Rehabilitacion (Madr). 2019, 53:104-110. 10.1016/j.rh.2018.04.007

3. Suga H, Araki J, Aoi N, Kato H, Higashino T, Yoshimura K: Adipose tissue remodeling in lipedema: adipocyte death and concurrent regeneration. J Cutan Pathol. 2009, 36:1293-1298. 10.1111/j.1600-0560.2009.01256.X

4. Amann-Vesti, BR, Franzeck UK, Bollinger A: Microlymphatic aneurysms in patients with lipedema . Lymphology. 2001, 34:170-175.

5. Bollinger A, Amann-Vesti BR: Fluorescence microlymphography: diagnostic potential in lymphedema and basis for the measurement of lymphatic pressure and flow velocity. Lymphology. 2007, 40:52-62.

6. Herbst KL, Mirkovskaya L, Bharhagava A, Chava Y, Te CH: Lipedema fat and signs and symptoms of illness, increase with advancing stage. Arch Med. 2015, 7:10.

7. Buso G, Depairon M, Tomson D, Raffoul W, Vettor R, Mazzolai L: Lipedema: a call to action!. Obesity. 2019, 27:1567-1576. 10.1002/oby.22597

8. Al-Ghadban S, Cromer W, Allen M, Ussery C, Badowski M, Harris D, Herbst KL: Dilated blood and lymphatic microvessels, angiogenesis, increased macrophages, and adipocyte hypertrophy in lipedema thigh skin and fat tissue. J Obes. 2019, 2019:8747461. 10.1155/2019/8747461

9. Pereira de Godoy JM, Barufi S, Guerreiro Godoy MF: Lipedema: is aesthetic cellulite an aggravating factor for limb perimeter?. J Cutan Aesthet Surg. 2013, 6:167-168. 10.4103/0974-2077.118431

10. Alwardat N, Di Renzo L, Alwardat M, et al.: The effect of lipedema on health-related quality of life and psychological status: a narrative review of the literature. Eat Weight Disord. 2020, 25:851-856. 10.1007/s40519-019-00703-x

11. Pereira de Godoy JM, Pereira de Godoy HJ, Guerreiro Godoy MF: Subclinical systemic lymphedema manifesting early in patients with clinical lymphedema. Int J Dev Res. 2018, 8:23041-23044.

12. Pereira de Godoy JM, Pereira de Godoy LM, Pereira de Godoy AC, Guerreiro Godoy MF: Bariatric surgery and the evaluation of subclinical systemic lymphedema. J Surg Case Rep. 2019, 2019:riz028. 10.1093/jscr/rjz028

13. Pereira de Godoy JM: Systemic subclinical lymphedema due to obesity as the cause of clinical lymphedema: a new concept. Med Hypotheses. 2019, 131:109312. 10.1016/j.mehy.2019.109312

14. Pereira de Godoy JM, Guerreiro Godoy MF: Diagnostic criteria and clinical evolution of systemic lymphedema caused by obesity: bioimpedance analysis. Ann Med Health Sci Res. 2019, 9:420-421.

15. Gould DJ, El-Sabawi B, Goel P, Badash I, Colletti P, Patel KM: Uncovering lymphatic transport abnormalities in patients with primary lipedema. J Reconstr Microsurg. 2020, 36:136-141. 10.1055/s-0039-1697904

16. Pereira de Godoy JM, Pereira de Godoy HJ, Pereira de Godoy LM, Guerreiro Godoy MF: Prevalence of idiopathic cyclic edema in women with lower limb lymphedema. J Clin Med. 2017, 7:2. 10.3390/jcm7010002

17. Crescenzi R, Marton A, Donahue PMC, et al.: Tissue sodium content is elevated in the skin and 


\section{Cureus}

subcutaneous adipose tissue in women with lipedema. Obesity. 2018, 26:310-317. 10.1002/oby.22090 